\title{
Paragangliom der Orbita - eine Rarität
}

\section{Einfihrung \\ $\nabla$}

Paragangliome, auch als Glomustumoren oder Chemodektome bezeichnet, entstammen den paraganglionären Zellen und sind Teil des extraadrenalen neuroendokrinen Systems. Paraganglien sind meist neben Nerven und Gefäßen lokalisiert und im ganzen Körper anzutreffen. Die vier häufigsten Lokalisationen im Kopf-Hals-Bereich umfassen die chemorezeptiven Areale entlang der Karotisbifurkation, das Foramen jugulare, den N. vagus und das Mittelohr.

Paragangliome können jedoch auch an anderen Stellen auftreten (z. B. Pinealisdrüse, Sella turcica, Sinus cavernosus, Orbita), stellen hier aber eine Rarität dar (Axmann C., Der Radiologe 2004; 4: 389 -400).

\section{Fallbeschreibung}

Ein 73-jähriger Patient wurde mit seit etwa drei Jahren bestehender Schwellung im Bereich der linken Orbita überwiesen. Die durchgeführte MRT zeigte in der linken Orbita intrakonal lateralseitig eine transversal $1,5 \times$ sagittal $1,3 \times \mathrm{kra}$ niokaudal $2,2 \mathrm{~cm}$ große, relativ glatt begrenzte Läsion mit starker Kontrast- mittelaufnahme und mit Verlagerung des $M$. rectus inferior nach medial, mit breitem Kontakt zum M. rectus lateralis sowie mit Heranreichen bis unmittelbar an den N. opticus. Nach kaudal reichte die Läsion bis an den Unterrand des Bulbus heran und imprimierte gering den linken Orbitaboden. Konsekutiv zeigte sich ein geringer Exophthalmus links. Die Läsion wurde als Hämangiom gewertet. Es zeigte sich eine unauffällige Darstellung der rechten Orbita sowie des Zerebrums. Von einer Operation wurde zunächst Abstand genommen.

Zwei Jahre später wurde der Patient erneut mit zunehmendem Exophthalmus und Doppelbildern beim Blick nach links und oben vorstellig.

Eine erneut durchgeführte MRT (beigefügte Abbildungen) zeigte eine Größenzunahme der deutlich kontrastmittelaufnehmenden Expansion ( $\bullet$ Abb. 1 ) intrakonal links mit nunmehr einer Größe von transversal 2,1 $\times$ sagittal 3,3 $\times$ kraniokaudal $3,4 \mathrm{~cm}$. Die Läsion zeigte im Inneren tubuläre signalarme Strukturen im Sinne von Flow voids ( $\bullet$ Abb.2) und imprimierte den Bulbus von dorsal, verlagerte den $\mathrm{M}$. rectus inferior nach medial, zeigte breiten Kontakt zum M. rectus lateralis und reichte bis unmittelbar an den N. opticus heran ( $\bullet$ Abb.3).

Unter dem Aspekt einer Histologiegewinnung und zur Tumorexstirpation wurde eine laterale Orbitotomie durchgeführt. Intraoperativ zeigte sich eine oberflächlich stark vaskularisierte Läsion mit immer wieder auftretenden diffusen Blutungen, welche zum Teil durch Koagulation, zum Teil durch Einlegen von Tachosilstückchen, gestillt werden konnten. Um den Sehnerven nicht zu gefährden, wurde von einer Radikalexstirpation des Tumors Abstand genommen.

Postoperativ kam es vorübergehend zu einer stärkeren Schwellung mit Verstärkung des Exophthalmus, dies bildete sich jedoch rasch unter Antibiose und Lokaltherapie zurück.

Die Histologie ( $\bullet$ Abb.4) sowie immunhistochemische Untersuchungen ergaben ein extraadrenales Paragangliom.

\section{Diskussion \\ $\nabla$}

Paragangliome sind äußerst seltene, stark vaskularisierte, größtenteils benigne Tumoren, die in bis zu 15\% ein malignes Wachstum zeigen (Axmann C., Der Radiologe 2004; 4: 389 -400).

Paragangliome der Orbita können sowohl Kinder als auch Erwachsene betreffen ohne Bevorzugung eines Geschlechtes. Die Patienten präsentieren sich mit Symptomen intraorbitaler Raumforderungen 


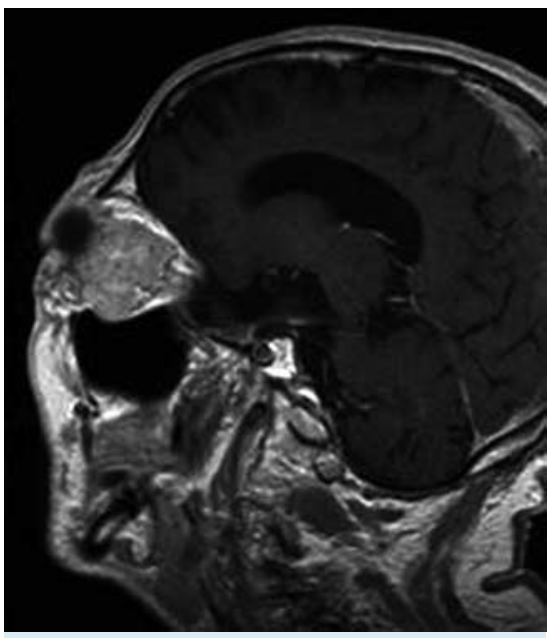

Abb.1 Deutliches und homogenes KM-Enhancement der Läsion. Impression des Orbitabodens. (SE $2 \mathrm{~d}, \mathrm{~T} 1 \mathrm{w}$ sagittal: TR $320 \mathrm{~ms}$, TE 7,7 ms; FOV $200 \mathrm{~mm}$, Schichtdicke $5 \mathrm{~mm}$; Applikation von $14 \mathrm{ml}$ Magnevist).

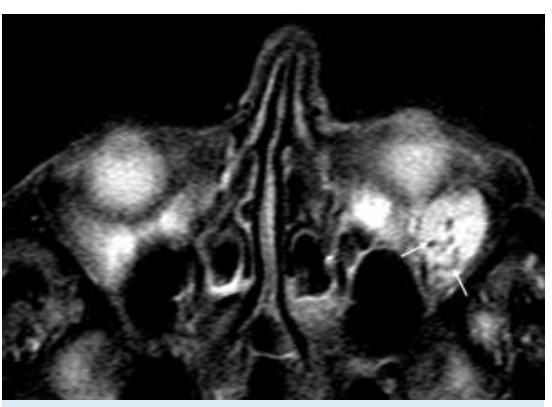

Abb.2 Paragangliom der linken Orbita mit tubulären signalarmen Strukturen (Flow voids; Pfeile). (TSE 2 d T $2 w$ transversal: TR $4020 \mathrm{~ms}$, TE 97 ms; FOV 230 mm, Schichtdicke $5 \mathrm{~mm}$ )

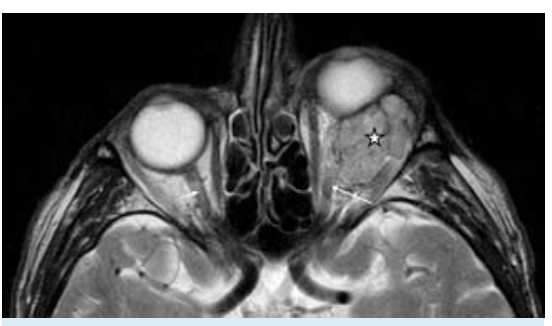

Abb. 3 Intrakonale hyperintense Raumforderung der linken Orbita (Stern) mit engem Kontakt zum N. opticus (langer Pfeil; kurzer Pfeil rechter N. opticus) und Impression des Bulbus von dorsal (TSE $2 \mathrm{~d}$ T $2 \mathrm{w}$ transversal: TR 4020 ms, TE 97 ms; FOV 230 mm, Schichtdicke $5 \mathrm{~mm}$ ).

wie Exophthalmus (kann aufgrund der starken Vaskularisation des Tumors pulsierend sein), Diplopie, reduziertem Sehvermögen, retrobulbären Schmerzen, geröteten Konjunktiven und Papillenödem. Es gibt keine Berichte über Symptome zu-

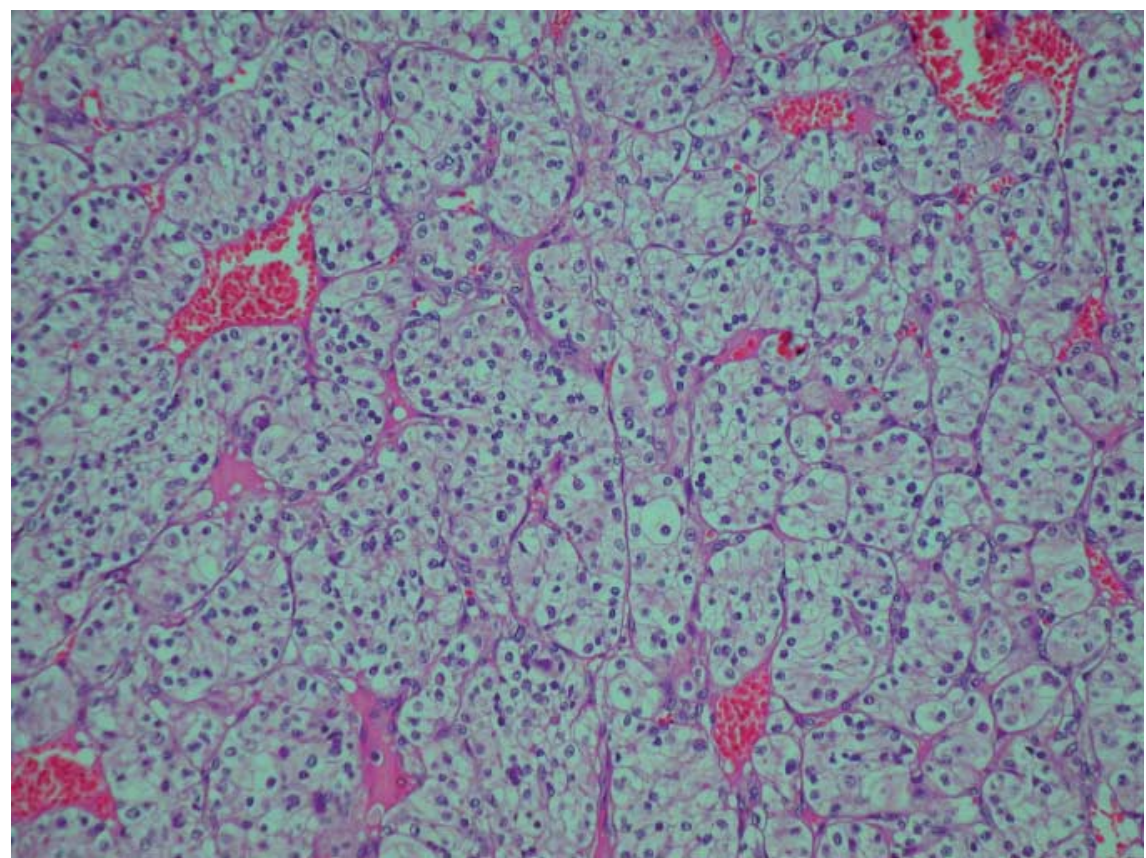

Abb.4 Das histologische Schnittbild aus dem Operationspräparat des Patienten zeigt ein typisches Bild eines Paraganglioms mit trabekulär oder alveolär angeordneten Tumorzellverbänden (sog. „Zellballen") und sinusoidalen Gefäßen (Haematoxylin - Eosin).

rückzuführen auf Katecholaminsekretion (Hypertonie, Tachykardie) von Paragangliomen in dieser Lokalisation.

Paragangliome imponieren in der Computertomografie als umschriebene, weichteildichte Raumforderungen, die aufgrund ihrer Hypervaskularität stark und homogen Kontrastmittel aufnehmen. Zudem sind in der CT knöcherne Erosionen und Infiltrationen gut zu differenzieren (Axmann C., Der Radiologe 2004; 4: 389-400).

In der MRT zeigen die Paragangliome in der T1- und Protonendichte-Wichtung ein hypo- bis isointenses Signalverhalten und sind in T2-Wichtung hyperintens. Typisch ist ein starkes und meist homogenes KM-Enhancement (Som PM et al., Radiology 1987; 164: 823 -829).

Ein Charakteristikum in der MRT-Bildgebung ist das in allen Wichtungen (v.a. in T2-Wichtung) häufig sichtbare „Salzund Pfeffer-Muster“. Dabei repräsentieren die dunklen „Pfefferanteile“ Flussauslöschungen (flow voids) und die „Salzanteile" kleine Tumoreinblutungen oder langsame Flussphänomene von tumorversorgenden Gefäßen (Olsen WL et al., AJR 1987; 148: 201 -204).

Der Tumor kann die Augenmuskeln verdrängen oder infiltrieren und den N. opticus umscheiden.

Paragangliome sind hypervaskularisierte Tumoren und zeigen dementsprechend in der Angiografie einen kräftigen und verzögerten Tumorblush. Die tumorversorgenden Gefäße sind in der Regel vergrößert, und ein früher venöser Abfluss resultiert durch AV-Shunts oder Fisteln (Weber AL et al., Neuroimag Clin North Am 1994; 4:579-598).

Differenzialdiagnostisch sind andere hypervaskularisierte Tumoren in Betracht zu ziehen, insbesondere Hämangiome, Hämangioendotheliome, Hämangioperizytome, Lymphangiome, Meningeome sowie Metastasen hypervaskularisierter Tumoren wie Hypernephrom, Schilddrüsenkarzinom und Melanom.

Die Therapie der Wahl ist die chirurgische Totalexzision des Tumors durch laterale Orbitotomie. Bei engem Kontakt zum N. opticus bzw. bei Umscheidung desselben wird eine Tumorteilexzision empfohlen (Sharma MC et al., Can J Ophthalmol 2005; 40: 640-644). Therapeutische Alternativen sind die Embolisation bzw. die Bestrahlung mittels Gammaknife.

Zusammenfassend sollten Paragangliome, obwohl in dieser Lokalisation selten, in die Differenzialdiagnose von hypervaskularisierten intraorbitalen Tumoren miteinbezogen werden.

B. Oberdabernig, H. Schoellnast, Graz 